\title{
Unconventional Internalization Mechanisms Underlying Functional Delivery of Antisense Oligonucleotides via Cationic Lipoplexes and Polyplexes
}

\author{
Xin Ming ${ }^{1}$, Katsuya Sato ${ }^{2}$, and Rudolph L. Juliano ${ }^{1}$ \\ ${ }^{1}$ Division of Molecular Pharmaceutics, UNC Eshelman School of Pharmacy, University of North \\ Carolina, Chapel Hill, NC 27599, USA \\ 2Department of Biomolecular Science, Faculty of Engineering, Gifu University, 1-1 Yanagido, Gifu \\ 501-1193, Japan
}

\begin{abstract}
There is mounting interest in developing antisense and siRNA oligonucleotides into therapeutic entities; however, this potential has been limited by poor access of oligonucleotides to their pharmacological targets within cells. Transfection reagents, such as cationic lipids and polymers, are commonly utilized to improve functional delivery of nucleic acids including oligonucleotides. Cellular entry of large plasmid DNA molecules with the assistance of these polycationic carriers is mediated by some form of endocytosis; however, the mechanism for delivery of small oligonucleotide molecules has not been well established. In this study, splice-shifting oligonucleotides have been formulated into cationic lipoplexes and polyplexes, and their internalization mechanisms have been examined by using pharmacological and genetic inhibitors of endocytosis. The results showed that intercellular distribution of the oligonucleotides to the nucleus governs their pharmacological response. A mechanistic study revealed that oligonucleotides delivered by lipoplexes enter the cells partially by membrane fusion and this mechanism accounts for the functional induction of the target gene. In contrast, polyplexes are internalized by unconventional endocytosis pathways that do not require dynamin or caveolin. These studies may help rationally design novel delivery systems with superior transfection efficiency but lower toxicity.
\end{abstract}

\section{Keywords}

Cellular delivery; Endocytosis; Internalization; Antisense oligonucleotides; Splice-shifting oligonucleotides; Intracellular trafficking; Cationic lipoplexes; Cationic polyplexes

\section{Introduction}

There is great potential for antisense and siRNA oligonucleotides to become mainstream therapeutic entities thanks to their high specificity and wide therapeutic target space as

(C) 2011 Elsevier B.V. All rights reserved.

Corresponding authors: Drs. Rudolph L. Juliano and Xin Ming, Division of Molecular Pharmaceutics, UNC Eshelman School of Pharmacy, 1072 Genetic Medicine Building, University of North Carolina at Chapel Hill, Chapel Hill, NC 27599, USA. arjay@med.unc.edu (RLJ) and xming@email.unc.edu (XM). Phone number: 1-919-966-4343.

Publisher's Disclaimer: This is a PDF file of an unedited manuscript that has been accepted for publication. As a service to our customers we are providing this early version of the manuscript. The manuscript will undergo copyediting, typesetting, and review of the resulting proof before it is published in its final citable form. Please note that during the production process errors may be discovered which could affect the content, and all legal disclaimers that apply to the journal pertain. 
compared to small molecules. However, the pharmacological targets within cells are poorly accessible to oligonucleotides that are hydrophilic and often charged macromolecules [1,2]. In order to reach these intracellular targets, therapeutic oligonucleotides must cross numerous biological barriers following administration, of which transport across the plasma membrane and then trafficking to the intracellular site of action have been generally considered as the rate-limiting steps [1-3]. One strategy to overcome these biological barriers and allow successful delivery is to formulate oligonucleotides into nanoparticles with cationic lipids and polymers. This strategy has achieved significant success in cellular and animal studies [4-7] and generated promising results in Phase I clinical studies in which therapeutic siRNAs were delivered to tumors by a liposomal formulation [8]. Recently, polymer-based nanoparticles coated with transferrin were shown to deliver siRNAs to solid tumors in humans and cause gene specific RNAi activity [9]; this is the first siRNA clinical trial that used a polymer-based delivery system.

To design even superior delivery systems for therapeutic oligonucleotides, it is necessary to understand how the cationic carriers assist internalization and trafficking of the oligonucleotides. On the other hand, toxicity is often associated with these types of carriers [10], and this can only be avoided by understanding the mechanisms that cause it. Using pharmacological inhibitors of endocytosis, the internalization mechanisms of plasmid DNA that is formulated with cationic lipids or polymers have been examined [11-13]. The results demonstrated that uptake of DOTAP/DNA lipoplexes is due to clathrin-mediated endocytosis, which is also responsible for the functional transfection [11, 12]. On the other hand, PEI/DNA polyplexes enter the cells via a combination of clathrin-mediated endocytosis and the caveolar pathway; however, only the latter pathway leads to effective endosomal escape and thereafter functional transfection [11-13]. Another study further demonstrated that plasmid DNAs formulated with different cationic lipids may undergo different endocytotic pathways [14]. The plasmid DNA complexed with DMRIE-C follows the caveolar pathway, while that formulated with Lipofectamine LTX is internalized by clathrin-mediated endocytosis [14]. Genetic inhibition of dynamin, which mediates pinching off the vesicles from the plasma membrane, reduces cellular uptake of both DNA lipoplexes dramatically [14]. Interestingly, a recent study has revealed a different mechanism for cellular entry of siRNAs delivered by lipoplexes [15]. The majority of siRNAs that are formulated in lipoplexes entered the cells by endocytosis, however, this pathway fails to produce functional knockdown, whereas the minor portion of the siRNAs, which are internalized possibly via membrane fusion, generates the target gene suppression [15]. This study highlighted that oligonucleotides may utilize a cellular entry mechanism that is different from that for larger DNA molecules, even when they are delivered with the same carrier system.

In the current study we have examined internalization mechanisms of antisense oligonucleotides formulated into cationic lipoplexes and polyplexes using pharmacological and genetic inhibitors of endocytosis. A 'splice-shifting oligonucleotide (SSO)', which is designed to correct splicing of an aberrant intron inserted into an eGFP reporter gene, has been formulated with a cationic lipid Lipofectamine $2000^{\circledR}$ (L2K) or a cationic polymer jetPEI $^{\mathrm{TM}}$ (PEI). Both are commonly used transfection reagents for plasmid DNA and oligonucleotides. Successful delivery of SSOs to the cell nucleus would lead to upregulation of eGFP expression, providing a positive read-out for functional delivery. This study showed that the SSOs delivered by lipoplexes enter the cells largely by membrane fusion and this mechanism also account for the functional induction of the target gene. On the other hand, PEI/SSO polyplexes are internalized by unconventional endocytosis pathways that do not require dynamin or caveolin functions. Preliminary study also demonstrated the different temperature dependence of functional oligonucleotide delivery via lipoplexes and polyplexes. 


\section{Materials and Methods}

\section{Synthesis and chemical characterization of SSOs}

The SSO (5'-GTTATTCTTTAGAATGGTGC-3') is 2'-O-Me oligonucleotide with phosphorothioate linkage. The SSO and its 3'-Tamra conjugate were prepared as previously reported [16]. In brief, oligonucleotides were synthesized using phosphoramidites of the ultraMILD-protected bases on CPG supports (Glen Research, Sterling, VA, USA) using a AB 3400 DNA synthesizer (Applied Biosystems, Foster City, CA, USA). After cleavage from the CPG support and deprotection, the oligonucleotides were purified by reverse-phase HPLC on a Varian HPLC system (ProStar/Dynamax, Walnut Creek, CA) and identified using MALDI-TOF mass spectroscopy on a Voyager Applied Biosystem instrument (Foster City, CA).

\section{Cell lines, plasmids, and transfection}

The Hela S3 cells, containing an aberrant intron inserted into the eGFP coding sequence, were a kind gift from Dr R. Kole (University of North Carolina) [17]. This stably transfected cell line was referred to as Hela/eGFP654 and was cultured in F12K medium (Invitrogen, Carlsbad, CA, USA) containing 10\% FBS (Sigma, St. Louis, MO) and $500 \mu \mathrm{g} / \mathrm{mlGeneticin}$ (Invitrogen). Human melanoma cells A375SM were cultured in DMEM (Invitrogen) plus $10 \%$ FBS. To construct A375SM cells expressing eGFP654, the eGFP654 cassette was cut from the plasmid pEGFP654 [17] at the BamH I and Not I site and inserted into pcDNA3.1(+)/hygro (Invitrogen) resulting in the plasmid pcDNA3.1/hygro/eGFP654. Stable transfectants were obtained by transfecting A375SM cells with pcDNA3.1/hygro/ eGFP654 plasmid using an Amaxa Nucleoporation system as per manufacturer's instructions. Selection was carried out in culture media containing $200 \mu \mathrm{g} / \mathrm{ml}$ hygromycin B (Roche) for 2 weeks. Individual clones were picked and screened for luciferase induction by the SSO complexed with L2K. The single cell clone with the highest expression induced by SSO was referred to as A375/eGFP654 and used in further studies.

The plasmids encoding eGFP-dynamin dominant negative (DN), RFP-dynamin DN, and RFP-caveolin DN were kindly provided by Dr. JoAnn Trejo (University of California at San Diego, USA), Dr. Jennifer Lippincott-Schwartz (National Institutes of Health, USA), and Dr. David Marks (Mayo Clinic and Foundation, USA), respectively. Plasmids expressing mutant dynamin or caveolin were transfected into the Hela/eGFP654 cells using an Amaxa Nucleoporation system as per manufacturer's instructions. Briefly, one million Hela/ eGFP654 cells were nucleofected using the Cell Line Nucleofector Kit V, program I-013, and $2 \mu \mathrm{g}$ of plasmids. The following day cells were treated with the oligonucleotide formulated with transfection reagents, and uptake and functional induction of the oligonucleotide were analyzed by flow cytometry, whereas their intracellular distribution was observed by confocal microscopy.

\section{Oligonucleotide transfection}

Hela/eGFP654 or A375/eGFP654 cells were seeded on 24-well plates at $5 \times 10^{4}$ cells per well in various experiments. The following day, cells were treated with the SSO complexed with either a cationic lipid L2K (Invitrogen) or a cationic polymer PEI (Polyplus, Illkirch, France) as per manufacturers' instructions. The amounts of the transfection reagents and the oligonucleotide were optimized to achieve considerable transfection efficiency (> 30\%). To achieve optimal transfection with the SSO lipoplexes, $1 \mu \mathrm{l}$ of L2K was diluted into $50 \mu \mathrm{l}$ of Opti-MEM I (Invitrogen). After 5-minute incubation at room temperature, the diluted L2K solution was then mixed gently with an equal volume of Opti-MEM I containing 30 pmoles of the SSO followed by 20-minute incubation at room temperature. The lipid/SSO complex was then added to each well containing $500 \mu \mathrm{l}$ Opti-MEM I. The resultant $0.6 \mathrm{ml}$ dose 
solution containing $50 \mathrm{nM} \mathrm{SSOs} \mathrm{was} \mathrm{incubated} \mathrm{with} \mathrm{cells} \mathrm{for} 4$ hours at $37^{\circ} \mathrm{C}$. The cells were subsequently washed with PBS, and then fresh culture media were added. Cells were cultured for another 24 hours before measuring the eGFP expression with flow cytometry.

The optimal transfection with the SSO polyplexes was achieved when the N/P ratio was 6.25. To prepare the polyplexes, $1 \mu \mathrm{l}$ of PEI was diluted into $50 \mu 1$ of $150 \mathrm{mM} \mathrm{NaCl}$ solution and was then mixed by vortex with an equal volume of $\mathrm{NaCl}$ solution containing 60 pmoles of the SSO. After 20-minute incubation at room temperature, the PEI/SSO polyplexes were then added to each well containing $500 \mu 1$ Opti-MEM I medium, which made up the $0.6 \mathrm{ml}$ dose solution containing $100 \mathrm{nM}$ SSO. Following the 4-hour treatment at $37^{\circ} \mathrm{C}$, the cells were subsequently washed with PBS and then replenished with fresh media for another 24hour culture. The functional delivery was examined by measuring the eGFP expression with flow cytometry.

Pharmacological inhibitors were used to identify possible endocytotic pathways and the following concentrations were selected based on the previous studies [16, 18, 19]: chlorpromazine, $25 \mu \mathrm{M}$; methyl- $\beta$-cyclodextrin (methyl- $\beta$-CD), $1 \mathrm{mM}$; amiloride, $100 \mu \mathrm{M}$; cytochalasin D, $2 \mu \mathrm{M}$. The cells were pretreated with the inhibitors for 30 minutes at $37^{\circ} \mathrm{C}$ and then transfected with lipoplexes or polyplexes for 4 hours at $37^{\circ} \mathrm{C}$ in the presence of the inhibitors. The cells were washed with PBS and cultured in fresh media in the absence of the inhibitors for another 24 hours prior to measuring eGFP expression with flow cytometry. In the experiment of temperature-dependent transfection, the cells were incubated at specified temperatures for 1 hour and then transfected with the oligonucleotide formulations at the same temperatures for 4 hours. The cells were washed, replenished with fresh media, and incubated for 24 hours at $37^{\circ} \mathrm{C}$ prior to functional assay. In the experiment of cellular uptake, the cells were treated for 2 hours at $37^{\circ} \mathrm{C}$ in the presence of the inhibitors, and then were trypsinized before evaluating the uptake with flow cytometry.

\section{Particle size and zeta potential measurement}

The average particle size of the lipoplexes in OPTI-MEM I and the polyplexes in $150 \mathrm{mM}$ $\mathrm{NaCl}$ solution was determined using a Coulter N5 Plus Sub-Micron Particle Sizer (Beckman Coulter, Miami, FL, USA) at a fixed angle of $90^{\circ}$ and a temperature of $25^{\circ} \mathrm{C}$. Light scattering intensity was maintained within the required range of the instrument $\left(5 \times 10^{4}\right.$ to 1 $\times 10^{6}$ counts/second) in all the measurements. Each sample was analyzed in triplicate. The same complexes were placed in the dip cell of Zetasizer Nano Z (Malvern Instruments, Westborough, MA, USA) to determine the zeta potential. Each sample was analyzed in quintuplicate.

\section{Flow cytometry and confocal fluorescence microscopy}

Total cellular uptake of the Tamra-labeled SSO and eGFP expression induced by the SSO were measured by flow cytometry using a LSR II cell analyzer (Becton-Dickenson, San Jose, CA, USA). After treatment with oligonucleotide formulations, the cells were trypsinized and analyzed by flow cytometry, with a $488 \mathrm{~nm}$ laser coupled with a 525/50 filter for eGFP and a $561 \mathrm{~nm}$ laser coupled with a 582/15 emission filter for both Tamra and RFP. In two-color flow cytometry, the cells containing a single fluorophore were used as control to set up compensation.

Intracellular distribution of the oligonucleotide in living cells was examined using a Zeiss 510 Meta Inverted Laser Scanning Confocal Microscope with 63×-oil immersion objectives (Carl Zeiss MicroImaging, Thornwood, NY, USA). After transfection of plasmids expressing eGFP tag, Hela/eGFP654 cells were plated in $35 \mathrm{~mm}$ glass bottom microwell dishes (MatTek, Ashland, MA), and then transfected with the oligonucleotide formulations. 
The cellular uptake of the SSO-Tamra was observed by confocal microscopy after 2-hour transfection, whereas the cellular accumulation of the SSO-Tamra and the eGFP induction was visualized after 4-hour transfection plus 24-hour culture.

\section{Data Analysis}

Data are expressed as mean \pm SD from three measurements unless otherwise noted.

Statistical significance was evaluated using ANOVA followed by Dunnet's test. The data were analyzed with GraphPad Prism 5 (GraphPad Software, Inc., La Jolla, CA).

\section{Results}

\section{Characterization of SSO complexes}

Average particle size and zeta potential values for the lipoplexes and polyplexes were summarized in Table 1. The average particle size of the lipoplexes was larger than the polyplexes. The SSO polyplexes showed positive Zeta potential, while the lipoplexes were negatively charged, which was consistent with the fact that plasmid DNA lipoplexes with the same lipid are negatively charged under optimum transfection condition [20].

\section{Functional delivery of SSO depends on its nuclear entry}

In order to examine the correlation between cellular uptake of the SSO complexes and their pharmacological responses, two-color flow cytometry was used to simultaneously measure uptake of Tamra labeled SSOs formulated with L2K or PEI (the abscissa) and the functional induction of eGFP reporter (the ordinate). The population of Hela/eGFP654 cells without any treatment (the control cells) only appeared in Q3 in the flow graph (data not shown). In Fig. 1A, the cell populations in Q2 and Q4 had higher Tamra fluorescence, indicating that SSO-Tamra was taken up by these cells; however, only the cells in Q2 expressed higher eGFP levels, indicating successful delivery of SSOs to their site of action in the nucleus.

Transfection with the SSOs formulated into lipoplexes or polyplexes led to functional eGFP induction due to correct splicing of an aberrant intron inserted into this reporter gene (Fig. 1A). The SSOs at $50 \mathrm{nM}$ complexed with $1 \mu 1$ of $\mathrm{L} 2 \mathrm{~K}$ produced $35 \%$ transfection efficiency (the percentage of the cells in $\mathrm{Q} 2$ versus the whole cell population) and the transfected cells (the cells in Q2 in Fig. 1A) showed 17-fold higher eGFP expression compared untransfected cells (those in Q4 in Fig. 1A). The SSOs at $100 \mathrm{nM}$ complexed with $1 \mu 1 \mathrm{PEI}(\mathrm{N} / \mathrm{P}=6.25$ ) produced similar transfection efficiency (30\%), and the transfected cells had 16-fold higher eGFP expression than untransfected cells (Fig. 1A). The results in Fig. 1A indicated that functional induction requires high accumulation of SSOs in the cells, since the cells with higher eGFP expression in Q2 also had higher accumulation of SSO-Tamra. On the other hand, in both L2K and PEI transfections, some cells in Q4 showed high SSO-Tamra accumulation; however it did not lead to eGFP induction, indicating that factors other than total uptake play important roles in governing effectiveness of the oligonucleotides. Compared to L2K lipoplexes, transfection with PEI/SSO polyplexes showed better correlation between cellular uptake and functional response, as more cells with high uptake of SSOs showed functional induction in polyplexes transfection (Fig. 1A).

In Fig. 1B, after transfection and following culture, Hela/eGFP654 cells were observed with confocal microscopy for the intracellular distribution of SSO-Tamra (shown in red) and functional eGFP expression (shown in green). The results, from both L2K and PEI transfection, demonstrated a strict correlation of nuclear entry of the oligonucleotide and functional eGFP induction (Fig. 1B). The cells with the SSO-Tamra in the nucleus (labeled with white arrows) showed strong eGFP expression, whereas the cells with the SSO-Tamra in the cytosol (labeled with blue arrows) failed to induce eGFP expression (Fig. 1B), 
indicating that the ability of oligonucleotides to traffic to the nucleus, the site of splicing correction, determines their effectiveness as therapeutic oligonucleotides.

\section{Pharmacological inhibition of functional lipoplex delivery}

Four pharmacological inhibitors were used in the study to examine the possible endocytosis pathways: chlorpromazine, an inhibitor of the clathrin pathway; methyl- $\beta-C D$, an inhibitor of the lipid raft mediated pathway by removing cholesterol from the plasma membrane; amiloride, an inhibitor of macropinocytosis; and cytochalasin D, an actin inhibitor. Treatment of methyl- $\beta$-CD reduced initial uptake of SSOs in lipoplexes in Hela/eGFP654 cells by $73 \%$ (Fig. 2A), which led to abolishment of the functional eGFP induction (over 93\% reduction in both expression level and transfection efficiency) (Fig. 2B). The SSOs were still accumulated in the vesicles in cytoplasm under methyl- $\beta-C D$ inhibition, however, they failed to produce any functional eGFP induction (Fig. 2D). The stronger effect on induction than on uptake can be explained by the notion that methyl- $\beta-C D$ only abolishes the functional uptake leading to nuclear entry of the SSOs as observed in the images in Fig. $2 \mathrm{D}$. In order to rule out the possibility that methyl- $\beta-\mathrm{CD}$ interrupted the formation of $\mathrm{L} 2 \mathrm{~K} /$ SSO lipoplexes when it is present in the transfection solution, the cells were treated with methyl- $\beta$-CD for 1 hour before the dosing of the lipoplexes. In Fig. $2 \mathrm{C}$, pretreatment of methyl- $\beta$-CD also reduced the functional eGFP induction by $80 \%$ even when it was not present together with the lipoplexes in the transfection step, indicating that the reduction of functional delivery by methyl- $\beta-C D$ was due to its direct interaction with the plasma membrane.

Chlorpromazine and cytochalasin D also reduced the uptake of lipoplex slightly (Fig. 2A); however, the reduction did not cause reduction in functional eGFP induction (Fig. 2B), indicating that the endocytosis pathways partially contribute to the total uptake of the lipoplexes, however, the SSOs in these pathways fail to reach the nucleus. This notion was supported by the observation in Fig. 2D showing that chlorpromazine did not reduce the nuclear entry of the oligonucleotide.

\section{Pharmacological inhibition of polyplex delivery}

The four pharmacological inhibitors were also used to examine the entry pathways of the $\mathrm{PEI} / \mathrm{SSO}$ polyplexes. Initial uptake of polyplex was reduced moderately by chlorpromazine (27\%) and methyl- $\beta-\mathrm{CD}(21 \%)$ (Fig. 3A), which produced moderate reduction in the functional eGFP induction (32\% and 28\% reduction in transfection efficiency, respectively) (Fig. 3B). Cytochalasin D did not affect the initial uptake of the polyplex, but reduced the transfection efficiency by $26 \%$.

\section{Functional delivery of SSOs by lipoplexes and polyplexes does not depend on dynamin function}

Since pharmacological inhibitors often have multiple effects we decided to further investigate the internalization pathways of the complexes by using more specific molecular inhibitors. Dynamin is a small GTPase that plays a key role in pinching off membrane vesicles and both clathrin-dependent endocytotic and caveolar pathways depend on the action of dynamin [21]. The potential role of dynamin in uptake of the complexes was examined by transfecting Hela/eGFP654 cells with plasmids coding for a chimeric protein comprised of a DN form of dynamin linked to eGFP. These cells were then treated with transferrin labeled with Alexa-594 or the complexes and observed for the extent of uptake of the fluorescent molecules. As seen in Fig. 4A, expression of high levels of DN-dynamineGFP almost completely blocked the accumulation of transferrin in intracellular vesicles, indicating that the function of dynamin was blocked in the transfected cells. However, the expression of DN-dynamin-eGFP failed to block the uptake of SSOs in lipoplexes or 
polyplexes as observed in the confocal images (Fig. 4A). In both cases, the SSO entered the nucleus of the cells expressing mutant dynamin, which could produce functional induction.

In order to directly assess the role of dynamin in effectiveness of the SSO delivered by lipoplexes and polyplexes, DN dynamin linked to RFP was expressed in Hela/eGFP654 cells. These cells were then treated with the lipoplexes or the polyplexes of the SSOs (without Tamra dye), and examined by two-color flow cytometry for expression of mutant dynamin (the abscissa) and functional expression of eGFP (the ordinate). After plasmid transfection, Hela/eGFP654 cells showed heterogeneous expression of mutant dynamin reflected by the pattern of RFP expression in flow. In Fig. 4B, the population of cells in Q2 and Q4 expressed DN-dynamin-RFP, while the population in Q1 and Q3 did not. Induction of eGFP by L2K/SSO or PEI/SSO complexes was similar between the mutant dynamin transfected and untransfected cells (Fig. 4B), confirming that functional delivery of SSOs by lipoplexes or polyplexes does not require dynamin function.

\section{Caveolin is not required in functional delivery of SSO via L2K or PEI complexes}

Caveolin is a scaffolding protein within caveolae membranes and plays a key role in the caveolar endocytosis pathway [22]. To examine whether caveolin plays a role in functional delivery of SSOs via by lipoplexes and polyplexes, DN caveolin linked to RFP was expressed into Hela/eGFP654 cells. These cells were then treated with the lipoplexes or the polyplexes of the SSO, and examined by flow cytometry for expression of mutant caveolin (the abscissa) and functional expression of eGFP (the ordinate). After plasmid transfection, Hela/eGFP654 cells showed heterogeneous expression of mutant caveolin reflected by the pattern of RFP expression in flow. In Fig. 5B, eGFP induction by L2K/SSO or PEI/SSO complexes was similar between the mutant caveolin transfected and untransfected cells, indicating that functional delivery of SSO by lipoplexes or polyplexes does not involve caveolin function.

\section{Temperature-dependent delivery of SSO via L2K or PEI complexes}

The effect of temperature on functional delivery of the SSO lipoplexes or polyplexes was examined by transfecting Hela/eGFP654 cells at $4,25,30$, or $37^{\circ} \mathrm{C}$. Functional eGFP induction by $\mathrm{PEI} / \mathrm{SSO}$ was greatly reduced at $4^{\circ} \mathrm{C}$ (less than $4 \%$ of the expression level compared to that at $37^{\circ} \mathrm{C}$ ), and the induction increased gradually until it reached a maximum at $37^{\circ} \mathrm{C}$ (Fig. 6A). In contrast, $\mathrm{L} 2 \mathrm{~K} / \mathrm{SSO}$ lipoplexes produced relatively high induction at $4^{\circ} \mathrm{C}$ (the expression level was over $40 \%$ of that at $37^{\circ} \mathrm{C}$ ), and the highest induction was achieved at $30^{\circ} \mathrm{C}$, which was 1.7 -fold higher than that at $37^{\circ} \mathrm{C}$ (Fig. 6A). Confocal imaging was performed to examine the intracellular distribution of the SSOs after the treatment of L2K/SSO-Tamra for 2 hours at these temperatures. The images in Fig. 6B showed that at $25^{\circ} \mathrm{C}$ and $30^{\circ} \mathrm{C}$, the SSO-Tamra delivered by $\mathrm{L} 2 \mathrm{~K}$ entered the nucleus in large amount within 2 hours.

\section{Pharmacological inhibition of functional delivery in A375/eGFP654 cells}

In order to test whether the novel internalization modes for non-viral delivery of SSOs are general mechanisms, A375/eGFP654 cells were treated with the same formulations of the SSO lipoplexes and polyplexes. Both SSO complexes produced abundant transfection in A375/eGFP654 cells, and the transfection efficiency were 39\% and 27\% for the SSO lipoplexes and polyplexes, respectively, which were similar to those obtained in Hela/ eGFP654 cells. The effects of pharmacological inhibitors on functional delivery of the SSOs in A375/eGFP654 cells were similar to those in Hela/eGFP654 cells (Fig. 7). For example, treatment of methyl- $\beta$-CD nearly abolished the functional eGFP induction by lipoplexes in both cell lines. However, treatment of chlorpromazine or cytochalasin $\mathrm{D}$ produced moderate reduction in functional eGFP induction in A375/eGFP654 cells (Fig. 7), indicating that the 
clathrin pathway partially contribute to the functional delivery of SSO lipoplexes in A375/ eGFP654 cells, but not in Hela/eGFP654 cells.

\section{Discussion}

This study began with elucidating how cellular uptake and intracellular distribution determine the pharmacological responses of oligonucleotides delivered via lipoplexes or polyplexes. In both delivery modes, high uptake is a prerequisite for the robust induction of the reporter gene, as indicated in Fig. 1A. However, uptake is not the only factor that governs the effectiveness of the oligonucleotides. Effective trafficking to the site of action, the nucleus in this case, is a more critical determinant, as observed in Fig. 1B. A previous study on lipoplex-based siRNA delivery showed that only 5\% of siRNAs that are delivered into cells by lipoplexes are responsible for the gene knockdown [15]. In contrast, the amount of the SSOs in the nucleus accounts for the greater part of the total intracellular oligonucleotides after both L2K/SSO and PEI/SSO transfections, as observed from the confocal images in Fig. 1B. This might indicate that cationic carriers may be superior delivery systems for single-strand antisense oligonucleotides compared to the siRNAs in terms of effective trafficking to intracellular action sites.

It is important to define the internalization pathways that lead to efficient functional delivery of oligonucleotides assisted by the cationic carriers. Lipoplexes of the oligonucleotides enter the cells via a combination of endocytotic and non-endocytotic pathways, and the latter process may occur through direct membrane fusion in the plasma membrane regions with high content of cholesterol. Clathrin and actin inhibitors did not affect the functional delivery of lipoplexes of the SSOs greatly, whereas methyl- $\beta-C D$, capable of removing cholesterol from the plasma membrane, blocked the nuclear entry of the SSOs, and abolished reporter induction (Fig. 2). Although cholesterol depletion can also disrupt caveolae-dependent endocytosis [23], genetic inhibition of caveolin and dynamin, both of which are required in caveolar pathway [24], failed to reduce the effectiveness of SSOs' lipoplexes (Figs. 4 and 5), which rules out the involvement of this pathway in functional delivery of SSOs via lipoplexes. Furthermore, lipoplex-based delivery of SSOs showed the best transfection at $30^{\circ} \mathrm{C}$ instead of $37^{\circ} \mathrm{C}$ (Fig. 6A), indicating this delivery may not be an active process such as endocytosis. In conclusion, the results in the current study support the concept that a membrane fusion mechanism is responsible for the functional delivery of antisense oligonucleotides via lipoplexes [15].

The study also demonstrated that the SSO polyplexes undergo an internalization pathway that is different from that for the lipoplexes. Methyl- $\beta-C D$ only reduced the uptake and effectiveness of PEI/SSO polyplexes moderately, so did the clathrin and actin inhibitors (Fig. 3). Genetic inhibition of dynamin or caveolin failed to diminish the effectiveness of SSO polyplexes (Figs. 4 and 5). Furthermore, functional delivery increased gradually with temperature until reaching a maximum at $37^{\circ} \mathrm{C}$ (Fig. 6), which is consistent with an active endocytosis process. Collectively, SSO polyplexes may arrive at the site of action via a combination of multiple noncaveolar and clathrin-independent pathways. This is the first study that demonstrated that cationic lipoplexes and polyplexes utilize different mechanisms to deliver oligonucleotides to their sites of action within cells. In both delivery modes, endocytosis contributes to the total cellular uptake of oligonucleotides, but only leads to functional delivery in PEI-mediated delivery. This may result from the effective endosomal release of the oligonucleotides in PEI polyplexes via the "proton sponge" effect [25], and this property may also lead to the superior correlation between cellular uptake and functional response for the delivery via PEI polyplexes (Fig. 1A). The internalization pathway of the $\mathrm{PEI} / \mathrm{SSO}$ polyplexes is also different from that of the PEI/DNA polyplexes, which largely depends on the caveolar pathway [11-13]. The SSO polyplexes have a mean particle size of 
$336 \mathrm{~nm}$, whereas PEI/DNA polyplexes are much larger with a particle size of about 1000 $\mathrm{nm}$ [13]. Therefore, the disparity may be due to the different sizes of the PEI polyplexes.

The finding that effectiveness of the SSO lipoplexes depends on direct membrane fusion rather than endocytosis suggests that internalization of nucleic acids complexed into lipoplexes largely depends on the sizes of the cargo molecules. Early studies have indicated that lipoplexes of plasmid DNA undergo some form of endocytosis, though the reports have disagreed on the exact endocytosis pathways [11, 12, 14]. More recently, correlation between membrane permeability and biological transfection of DNA lipoplexes and polyplexes was examined by using two-color flow cytometry [26]. It has been found that cell membrane porosity caused by polycationic carriers does not enhance functional delivery of DNA and is actually an undesired side effect that limits transfection efficiency [26]. On the other hand, a study on lipoplex-based siRNA delivery supported a non-endocytotic mechanism for functional delivery of smaller oligonucleotide molecules [15]; this is further supported by the current study. This disparity may result from the fact that the size of the pores created in membrane fusion is only big enough for smaller oligonucleotides to pass. Biophysical methods are needed to examine the physical processes of the membrane fusion involving functional delivery of oligonucleotides.

Direct membrane fusion as an internalization mechanism may benefit the functional delivery of oligonucleotides because this may avoid the endosomal release step, the rate-limiting step for many gene and siRNA delivery strategies [27]. Whether the oligonucleotides are still coupled with delivery reagents after internalization is still unknown. However, extensive nuclear staining after treatment of SSO lipoplexes was observed within an hour (data not shown) and nuclear entry of whole lipoplexes is unlikely because of the size-limited entry through nuclear pores [28]. This evidence favors the notion that the oligonucleotides are free of the lipids after cellular uptake. On the other hand, nuclear entry of the SSOs delivered by PEI polyplexes was much slower compared to L2K/SSO (data not show), which possibly indicated that PEI/SSO entry is mediated by endocytosis which requires an extra step of endosomal release. In spite of the slow onset, a large part of the oligonucleotides delivered by PEI was released from the endosome and accumulated in the nucleus after 24-hour culture as observed in Fig. 1B, possibly via the "proton sponge" effect [25].

In conclusion, this study showed polycationic carriers are suitable delivery systems for cellular delivery of antisense oligonucleotides. The mechanistic study revealed that the SSOs delivered by lipoplexes enter the cells largely by membrane fusion and this mechanism also account for the functional induction of the target gene, whereas PEI/SSO polyplexes are internalized by unconventional endocytosis pathways that do not require dynamin and caveolin functions. These mechanisms may help rationally design novel delivery systems and also help understand the toxicity associated with these delivery systems.

\section{Supplementary Material}

Refer to Web version on PubMed Central for supplementary material.

\section{Abbreviations}

$\begin{array}{ll}\text { SSO } & \text { splice-shifting oligonucleotide } \\ \text { eGFP } & \text { enhanced green fluorescence protein } \\ \text { RFP } & \text { red fluorescence protein }\end{array}$



DN dominant negative
L2K Lipofectamine $2000^{\circledR}$
PEI Polyethyleneimine

\section{Acknowledgments}

We gratefully acknowledge Dr. Ryszard Kole (University of North Carolina at Chapel Hill, USA) for providing the plasmid of pEGFP654 and Hela/eGFP654 cell line, Dr. JoAnn Trejo (University of California at San Diego, USA) for providing the plasmid of eGFP-DN-dynamin, Dr. Jennifer Lippincott-Schwartz (National Institutes of Health, USA) for providing the plasmid of RFP-dynamin DN, and Dr. David Marks (Mayo Clinic and Foundation, USA) for providing the plasmid of RFP-caveolin DN. We thank Lan Feng (University of North Carolina at Chapel Hill, USA) for the assistance with physical characterization of the SSO complexes. This work was supported by grant P01GM059299 to RLJ.

\section{References}

1. Juliano R, Bauman J, Kang H, Ming X. Biological barriers to therapy with antisense and siRNA oligonucleotides. Mol Pharm. 2009; 6(3):686-695. [PubMed: 19397332]

2. Whitehead KA, Langer R, Anderson DG. Knocking down barriers: advances in siRNA delivery. Nat Rev Drug Discov. 2009; 8(2):129-138. [PubMed: 19180106]

3. Ming X. Cellular delivery of siRNA and antisense oligonucleotides via receptor-mediated endocytosis. Expert Opin Drug Deliv. 2011; 8(4):435-449. [PubMed: 21381985]

4. Gao K, Huang L. Nonviral methods for siRNA delivery. Mol Pharm. 2009; 6(3):651-658. [PubMed: 19115957]

5. Wu SY, McMillan NA. Lipidic systems for in vivo siRNA delivery. AAPS J. 2009; 11(4):639-652. [PubMed: 19757082]

6. Zimmermann TS, Lee AC, Akinc A, Bramlage B, Bumcrot D, Fedoruk MN, Harborth J, Heyes JA, Jeffs LB, John M, Judge AD, Lam K, McClintock K, Nechev LV, Palmer LR, Racie T, Rohl I, Seiffert S, Shanmugam S, Sood V, Soutschek J, Toudjarska I, Wheat AJ, Yaworski E, Zedalis W, Koteliansky V, Manoharan M, Vornlocher HP, MacLachlan I. RNAi-mediated gene silencing in non-human primates. Nature. 2006; 441(7089):111-114. [PubMed: 16565705]

7. Frank-Kamenetsky M, Grefhorst A, Anderson NN, Racie TS, Bramlage B, Akinc A, Butler D, Charisse K, Dorkin R, Fan Y, Gamba-Vitalo C, Hadwiger P, Jayaraman M, John M, Jayaprakash KN, Maier M, Nechev L, Rajeev KG, Read T, Rohl I, Soutschek J, Tan P, Wong J, Wang G, Zimmermann T, de Fougerolles A, Vornlocher HP, Langer R, Anderson DG, Manoharan M, Koteliansky V, Horton JD, Fitzgerald K. Therapeutic RNAi targeting PCSK9 acutely lowers plasma cholesterol in rodents and LDL cholesterol in nonhuman primates. Proceedings of the National Academy of Sciences of the United States of America. 2008; 105(33):11915-11920. [PubMed: 18695239]

8. Vaishnaw AK, Gollob J, Gamba-Vitalo C, Hutabarat R, Sah D, Meyers R, de Fougerolles T, Maraganore J. A status report on RNAi therapeutics. Silence. 2010; 1(1):14. [PubMed: 20615220]

9. Davis ME, Zuckerman JE, Choi CH, Seligson D, Tolcher A, Alabi CA, Yen Y, Heidel JD, Ribas A. Evidence of RNAi in humans from systemically administered siRNA via targeted nanoparticles. Nature. 2010; 464(7291):1067-1070. [PubMed: 20305636]

10. Lv H, Zhang S, Wang B, Cui S, Yan J. Toxicity of cationic lipids and cationic polymers in gene delivery. J Control Release. 2006; 114(1):100-109. [PubMed: 16831482]

11. Rejman J, Bragonzi A, Conese M. Role of clathrin- and caveolae-mediated endocytosis in gene transfer mediated by lipo- and polyplexes. Mol Ther. 2005; 12(3):468-474. [PubMed: 15963763]

12. Rejman J, Conese M, Hoekstra D. Gene transfer by means of lipo- and polyplexes: role of clathrin and caveolae-mediated endocytosis. J Liposome Res. 2006; 16(3):237-247. [PubMed: 16952878]

13. van der Aa MA, Huth US, Hafele SY, Schubert R, Oosting RS, Mastrobattista E, Hennink WE, Peschka-Suss R, Koning GA, Crommelin DJ. Cellular uptake of cationic polymer-DNA 
complexes via caveolae plays a pivotal role in gene transfection in COS-7 cells. Pharm Res. 2007; 24(8):1590-1598. [PubMed: 17385010]

14. Wong AW, Scales SJ, Reilly DE. DNA internalized via caveolae requires microtubule-dependent, Rab7-independent transport to the late endocytic pathway for delivery to the nucleus. J Biol Chem. 2007; 282(31):22953-22963. [PubMed: 17562704]

15. Lu JJ, Langer R, Chen J. A novel mechanism is involved in cationic lipid-mediated functional siRNA delivery. Mol Pharm. 2009; 6(3):763-771. [PubMed: 19292453]

16. Ming X, Alam MR, Fisher M, Yan Y, Chen X, Juliano RL. Intracellular delivery of an antisense oligonucleotide via endocytosis of a $G$ protein-coupled receptor. Nucleic Acids Res. 2010; 38(19): 6567-6576. [PubMed: 20551131]

17. Sazani P, Kang SH, Maier MA, Wei C, Dillman J, Summerton J, Manoharan M, Kole R. Nuclear antisense effects of neutral, anionic and cationic oligonucleotide analogs. Nucleic Acids Res. 2001; 29(19):3965-3974. [PubMed: 11574678]

18. Alam MR, Dixit V, Kang H, Li ZB, Chen X, Trejo J, Fisher M, Juliano RL. Intracellular delivery of an anionic antisense oligonucleotide via receptor-mediated endocytosis. Nucleic Acids Res. 2008; 36(8):2764-2776. [PubMed: 18367474]

19. Alam MR, Ming X, Dixit V, Fisher M, Chen X, Juliano RL. The biological effect of an antisense oligonucleotide depends on its route of endocytosis and trafficking. Oligonucleotides. 2010; 20(2): 103-109. [PubMed: 20038250]

20. Son KK, Patel DH, Tkach D, Park A. Cationic liposome and plasmid DNA complexes formed in serum-free medium under optimum transfection condition are negatively charged. Biochim Biophys Acta. 2000; 1466(1-2):11-15. [PubMed: 10825426]

21. Robinson MS. The role of clathrin, adaptors and dynamin in endocytosis. Current opinion in cell biology. 1994; 6(4):538-544. [PubMed: 7986531]

22. Liu P, Rudick M, Anderson RG. Multiple functions of caveolin-1. J Biol Chem. 2002; 277(44): 41295-41298. [PubMed: 12189159]

23. Parpal S, Karlsson M, Thorn H, Stralfors P. Cholesterol depletion disrupts caveolae and insulin receptor signaling for metabolic control via insulin receptor substrate-1, but not for mitogenactivated protein kinase control. J Biol Chem. 2001; 276(13):9670-9678. [PubMed: 11121405]

24. Conner SD, Schmid SL. Regulated portals of entry into the cell. Nature. 2003; 422(6927):37-44. [PubMed: 12621426]

25. Boussif O, Lezoualc'h F, Zanta MA, Mergny MD, Scherman D, Demeneix B, Behr JP. A versatile vector for gene and oligonucleotide transfer into cells in culture and in vivo: polyethylenimine. Proc Natl Acad Sci U S A. 1995; 92(16):7297-7301. [PubMed: 7638184]

26. Prevette LE, Mullen DG, Holl MM. Polycation-induced cell membrane permeability does not enhance cellular uptake or expression efficiency of delivered DNA. Mol Pharm. 2010; 7(3):870883. [PubMed: 20349965]

27. Dominska M, Dykxhoorn DM. Breaking down the barriers: siRNA delivery and endosome escape. J Cell Sci. 2010; 123(Pt 8):1183-1189. [PubMed: 20356929]

28. Dean DA, Strong DD, Zimmer WE. Nuclear entry of nonviral vectors. Gene Ther. 2005; 12(11): 881-890. [PubMed: 15908994] 
A

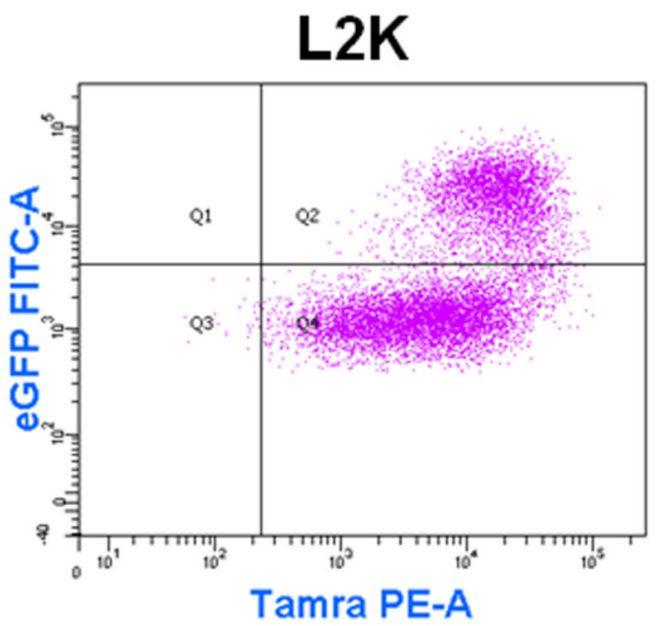

PEI

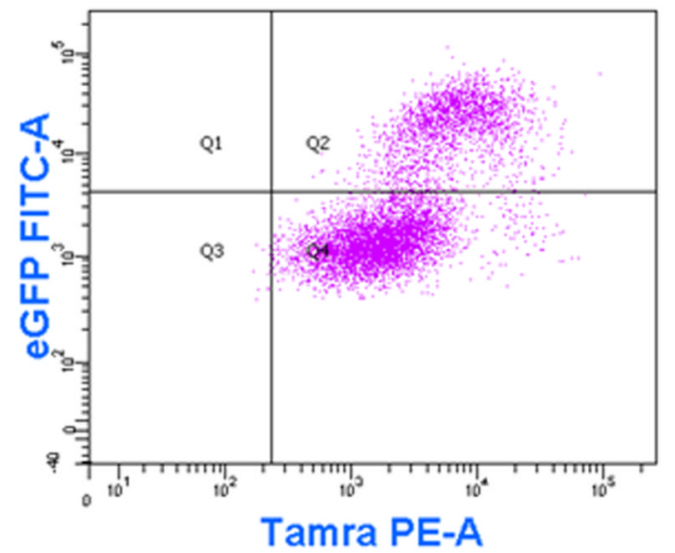

B

SSO-Tamra
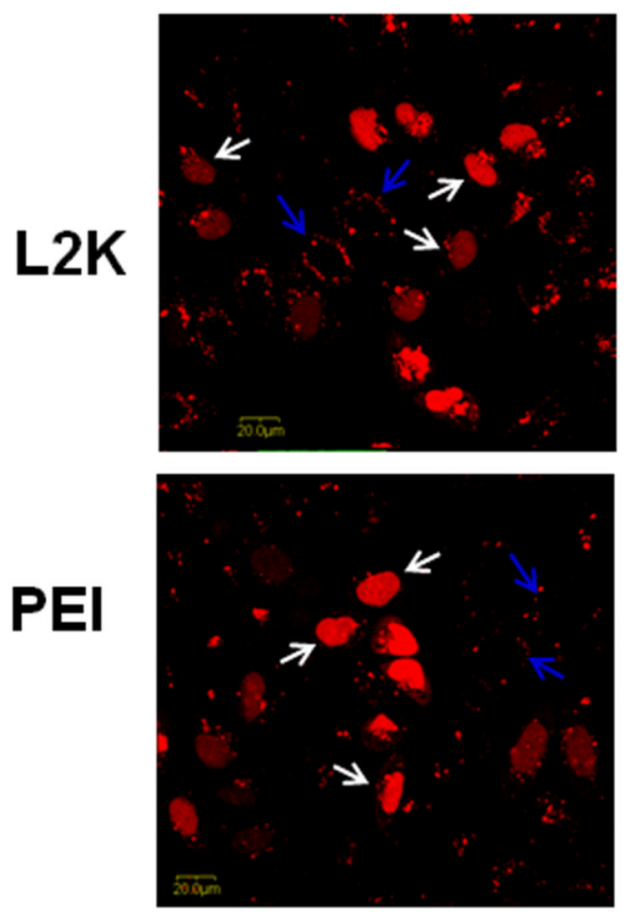

eGFP
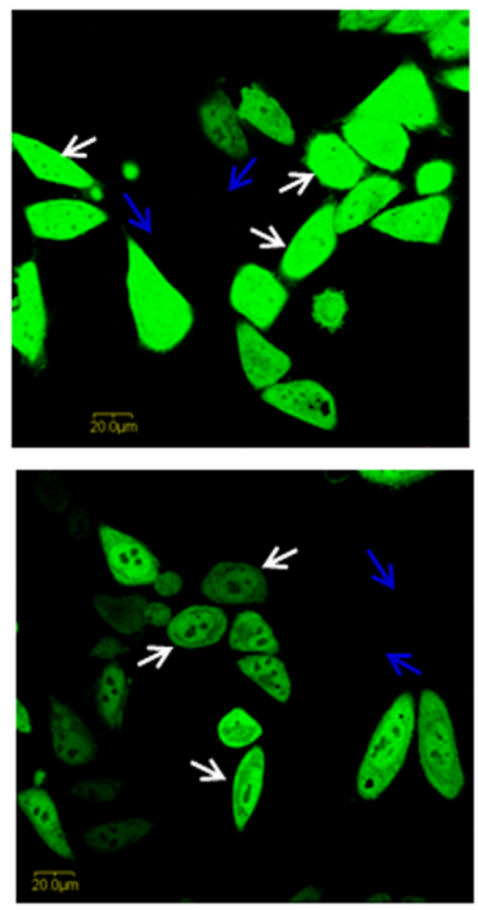

Merge
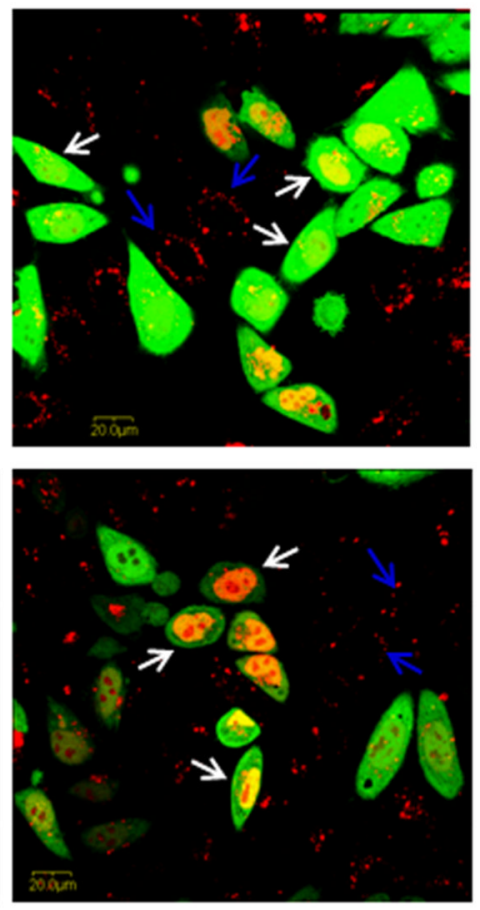

Fig. 1.

Relationship between cellular uptake and functional induction of SSOs in Hela/eGFP654 cells. After 4-hour transfection followed by 24-hour culture, the accumulation of SSOTamra (the abscissa) and induction of eGFP (the ordinate) in Hela/eGFP654 cells were analyzed with flow cytometry (A), and the cellular distribution of SSO-Tamra (red) and induction of eGFP (green) were observed with a confocal microscope (B). 
A. Uptake

Fig. 2.

\section{Cellular distribution sSO-Tamra}
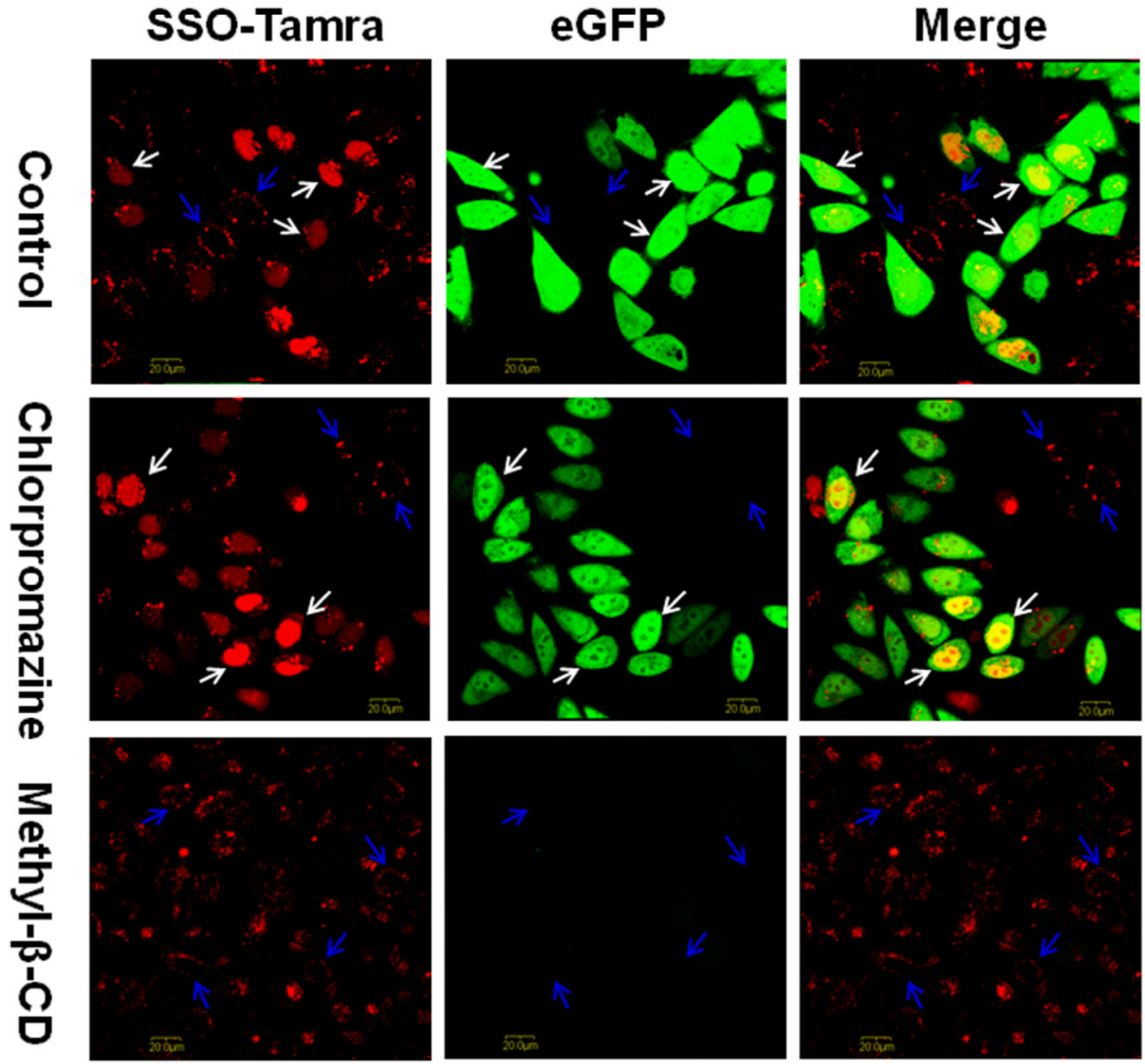

Effects of pharmacological inhibitors on uptake (A), functional induction (B and C), and cellular distribution (D) of SSOs delivered to Hela/eGFP654 cells by L2K lipoplexes. C. After pre-incubation with methyl- $\beta$-CD for 1 hour, Hela/eGFP654 cells were treated with L2K/SSO-Tamra lipoplexes in the presence (Co-treated) or the absence (Pre-treated) of the inhibitor for 4 hours, the eGFP induction was measured by flow cytometry after 24 hours. In $\mathrm{A}-\mathrm{C}$, the results are normalized based on cells receiving no inhibitor (Control) as $100 \%$ and represent means and standard deviations of triplicate determinations. 
A. Uptake

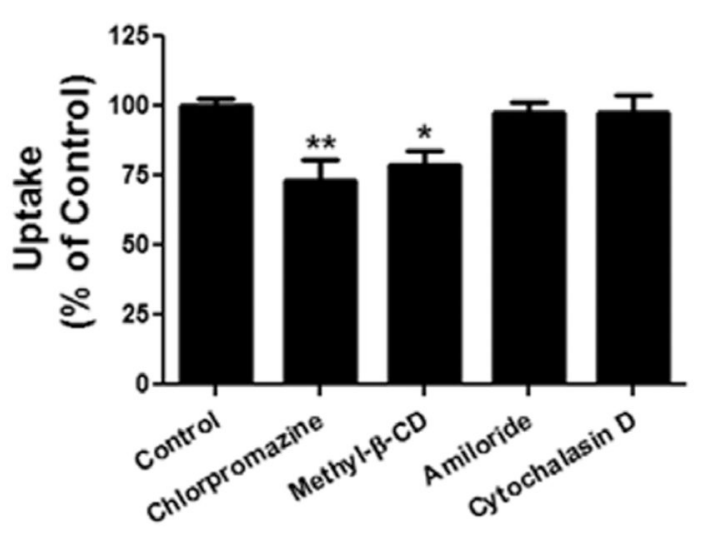

B. Induction

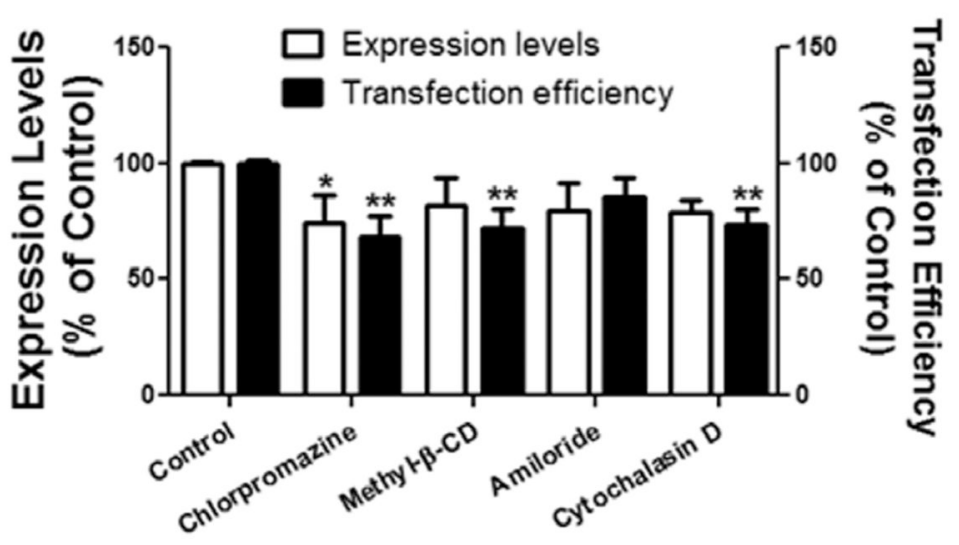

Fig. 3.

Effects of pharmacological inhibitors on uptake (A) and functional induction (B) in Hela/ eGFP654 by PEI polyplexes. Results are normalized on cells receiving no inhibitor (Control) as $100 \%$ and represent means and standard deviations of triplicate determinations. $* * * p<0.001, * * p<0.01$ and $* p<0.05$ compared with control. 


\section{A. Uptake}

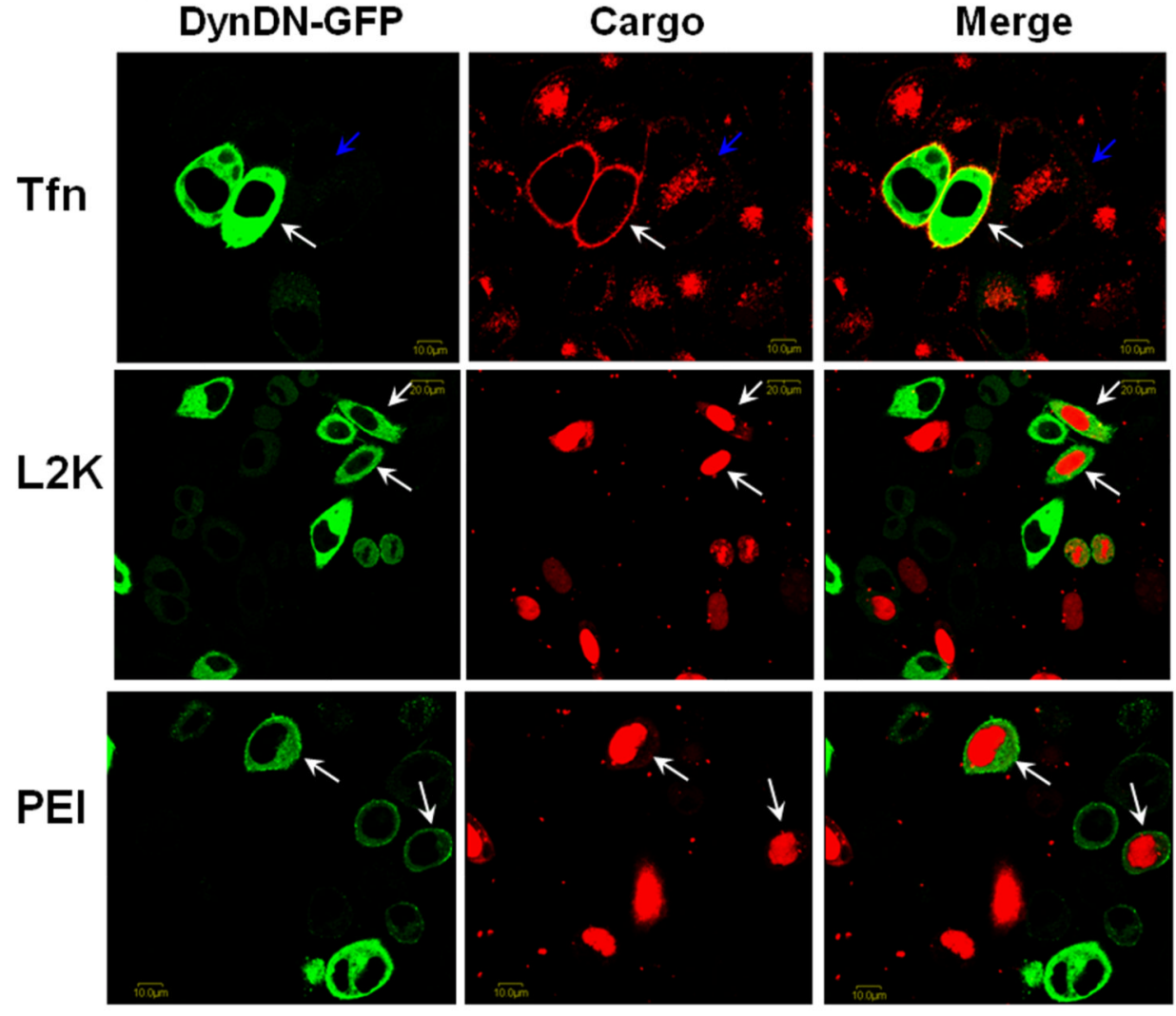

\section{B. Induction}
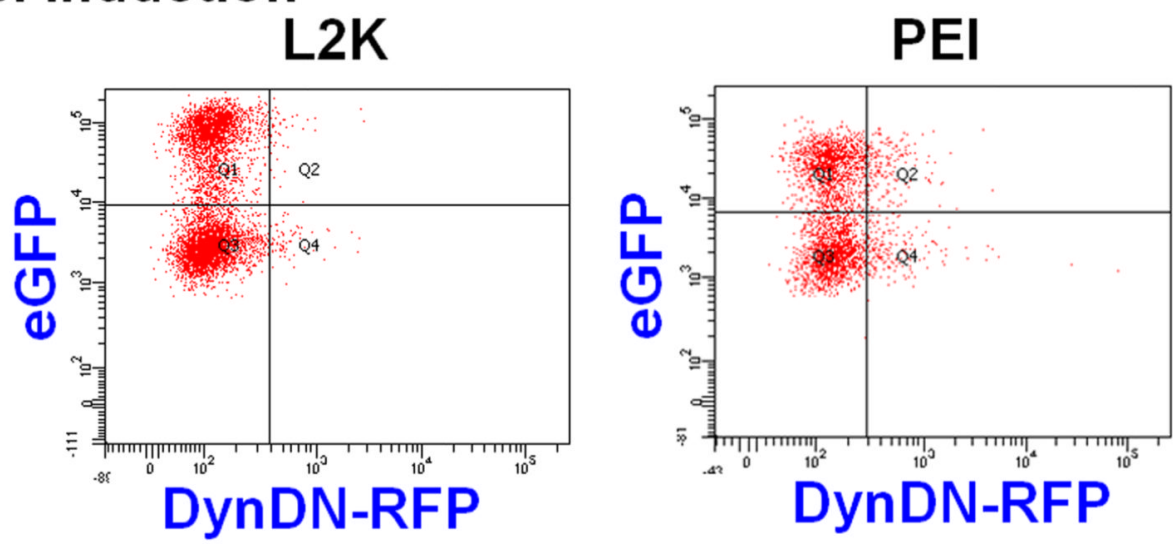

Fig. 4.

Effects of DN dynamin on cellular uptake (A) and functional induction (B) by SSO lipoplexes or polyplexes. A. Hela/eGFP654 cells were transfected with DN-dynamin-eGFP, and then were treated with transferrin-Alexa-594, the lipoplexes or the polyplexes of SSOTamra, and observed by confocal microscopy. B. Hela/eGFP654 cells were transfected with DN-dynamin-RFP. These cells were then treated with the lipoplexes or the polyplexes of the SSOs, and examined by two-color flow cytometry for expression of mutant dynamin (the abscissa) and functional expression of eGFP (the ordinate). 

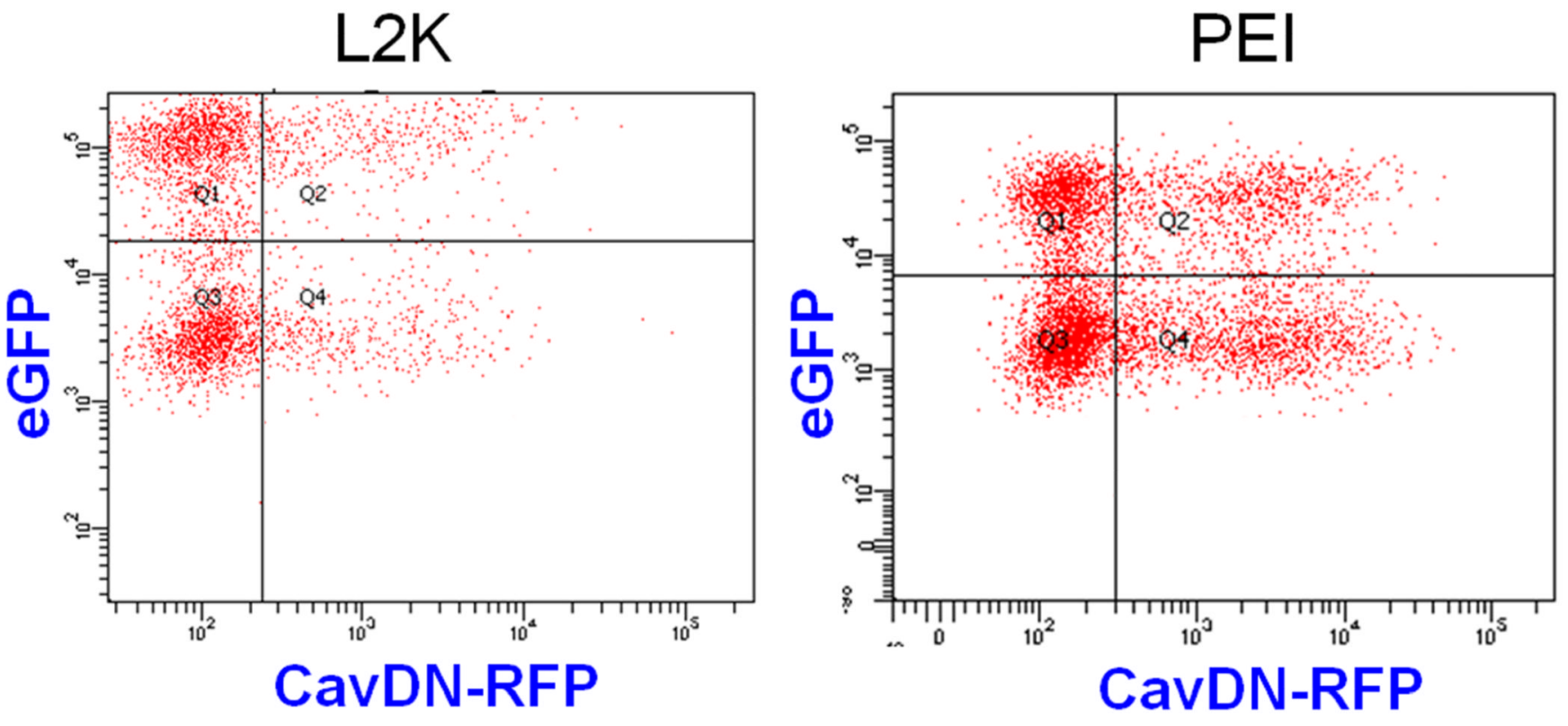

Fig. 5.

Effects of DN caveolin on functional induction of SSO lipoplexes or polyplexes. Hela/ eGFP654 cells were transfected with DN-caveolin-RFP, and were then treated with the lipoplexes or the polyplexes of the SSOs, and examined by two-color flow cytometry for expression of mutant caveolin (the abscissa) and functional expression of eGFP (the ordinate). 


\section{A. Induction}
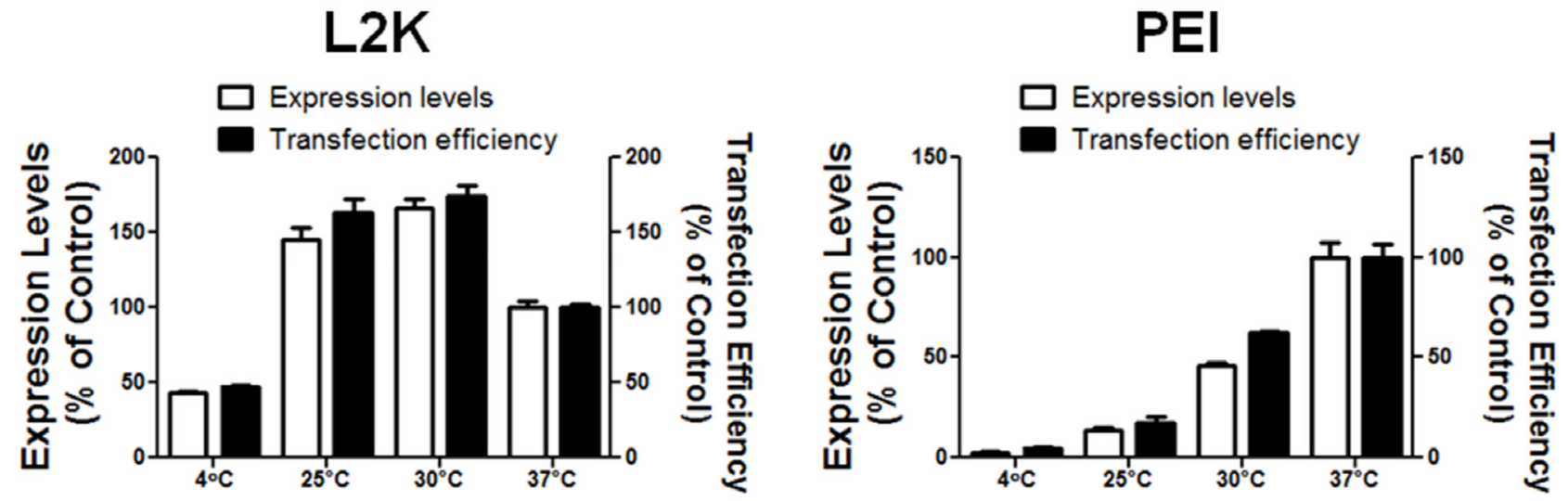

\section{B. Uptake (L2K)}

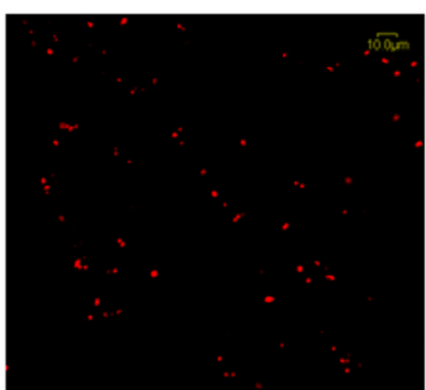

$4^{\circ} \mathrm{C}$

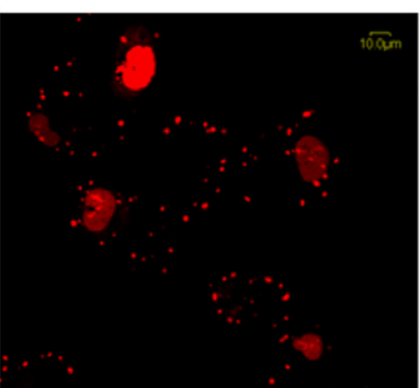

$25^{\circ} \mathrm{C}$

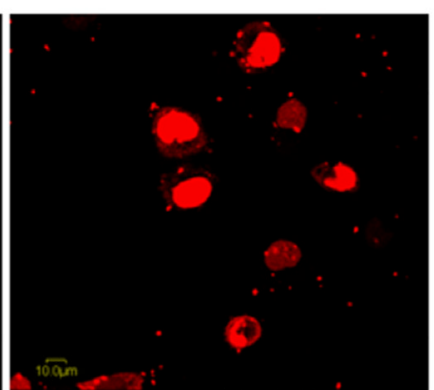

$30^{\circ} \mathrm{C}$

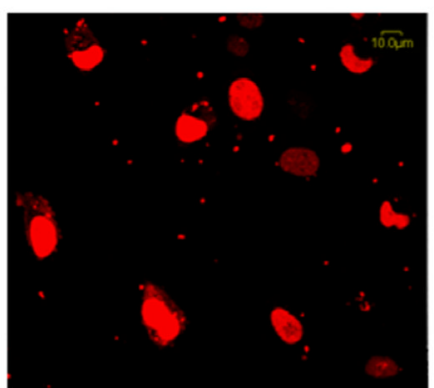

$37^{\circ} \mathrm{C}$

Fig. 6.

Effects of temperature on functional induction of the SSO lipoplexes and polyplexes. A. Hela/eGFP654 cells were transfected at the indicted temperatures, and the induction was measured with flow cytometry. Results are normalized based on cells treated at $37^{\circ} \mathrm{C}$ as $100 \%$ and represent means and standard deviations of triplicate determinations. B. After transfected for 2 hours at specified temperatures, Hela/eGFP654 cells were washed and observed by confocal microscopy for the extent and subcellular distribution of the fluorescent molecules. 
L2K

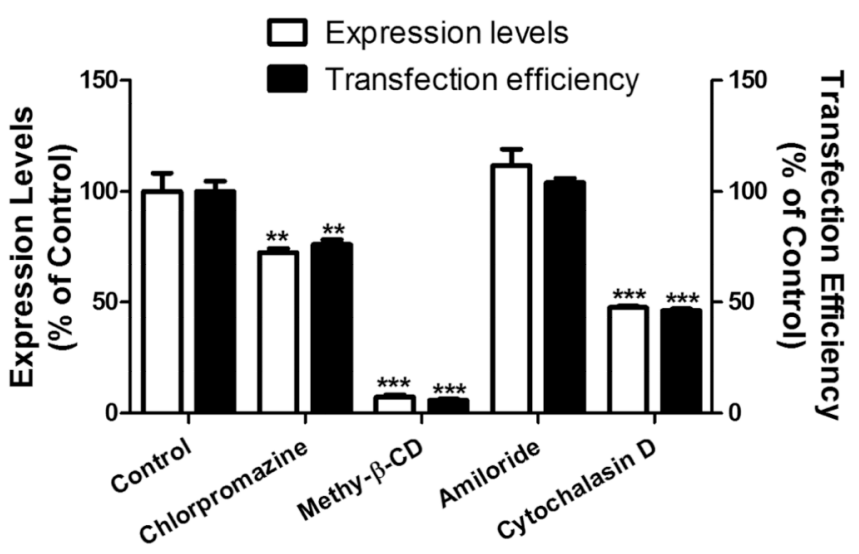

PEI

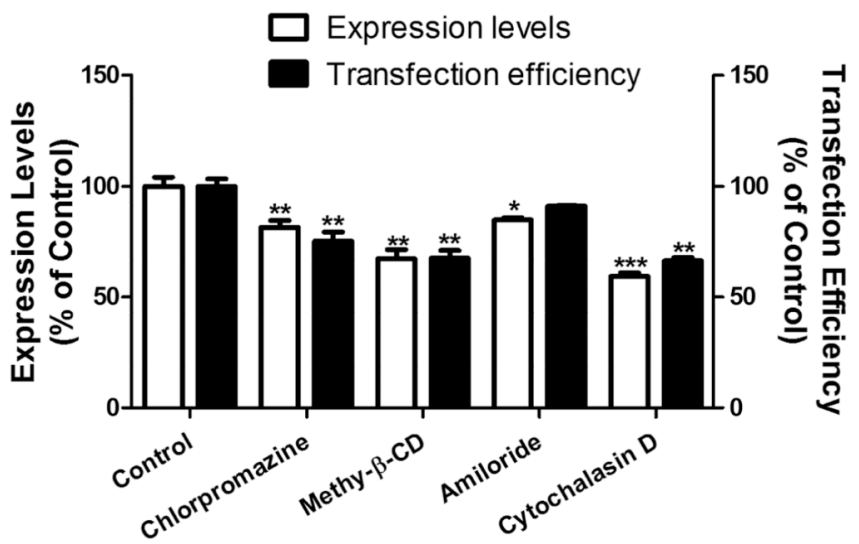

Fig. 7.

Effects of pharmacological inhibitors on functional induction by L2K lipoplexes (A) and PEI polyplexes (B) in A375/eGFP654 cells. Results are normalized based on cells receiving no inhibitor (Control group) as 100\% and represent means and standard deviations of triplicate determinations. ${ }^{* * *} p<0.001, * * p<0.01$ and $* p<0.05$ compared with control. 
Table 1

Characterization of lipoplexes and polyplexes. Results are expressed as mean \pm SD $(n=3-5)$

\begin{tabular}{lcc}
\hline & Particle Size (nm) & Zeta Potential $(\mathbf{m V})$ \\
\hline Lipoplexes & $869.6 \pm 83.0$ & $-28.8 \pm 2.4$ \\
Polyplexes & $335.6 \pm 5.9$ & $25.8 \pm 1.5$ \\
\hline
\end{tabular}

